\title{
Presencia en la Red de las Televisiones Parlamentarias en España
}

\author{
Ana Terroso Conejero \\ Jorge Caldera-Serrano
}

\section{Resumen}

Se analizan las expresiones web de las televisiones de las asambleas y parlamentos autonómicos (además de las cortes y el senado) de España, con el fin de verificar en un primer momento su existencia como su forma de difusión, ya sea por medios propios presentes en la red como por otros mecanismos o terceros canales como la utilización de canales de Youtube como plataforma de difusión. No sólo se analiza la presencia o existencia de dichos canales sino el formato de difusión así como la existencia de elementos propios de un sistema de conservación y almacenamiento documental como la existencia de videotecas, posibilidades de consultas e histórico de documentos audiovisuales. Como estado de la cuestión se enumeran la existencia de diferentes experiencias de televisiones parlamentarias a nivel mundial, así como se ofrece un repaso rápido por la evolución de los tipos televisiones.

\section{Palabras claves}

Televisiones parlamentarias, España, Internet, Transparencia informativa, Paradigma digital, Producción Audiovisual

\section{Presence in the television network in Spain parliamentary}

\section{Abstract}

Web expressions televisions assemblies and regional parliaments (besides the courts and the senate) of Spain, in order to verify at first its existence and form of distribution, either by own present analyzes the Net or by other mechanisms or third channels as using Youtube as channels broadcasting platform. Not only analyzes the presence or existence of such channels, but the broadcast format and the existence of elements of a conservation system and document storage as the existence of video libraries, and historical queries possibilities of audiovisual documents. As a state of the existence of different experiences of parliamentary televisions worldwide are listed as well as a quick run through the evolution of television types offered.

\section{Keywords}

Parliamentary Television, Spain, Internet, Information transparency, Digital paradigm, Audiovisual Production

http://dx.doi.org/10.5209/rev_CDMU.2013.v24.46278 


\section{INTRODUCCIÓN}

Crisis política, crisis institucional, crisis de valores. Los dirigentes políticos se encuentran en estos momentos entre las preocupaciones más importantes en un país como España, con una alta tasa de paro, con un número creciente de personas por debajo del umbral de la pobreza, con una valoración personal e institucional históricamente baja.

Son muchos los mecanismos que los dirigentes políticos han realizado para cambiar esta visión que la sociedad tiene de la "casta" política, de unos dirigentes criticados, y también poco entendidos, y manchados por grandes escándalos, independientemente de ser ajenos a ellos.

La política ha utilizado el marketing personal e institucional para avalar y mejorar las expectativas electorales por medio de la mejora de la imagen corporativa y personal de sus líderes. Y las instituciones también han debido llevar a cabo este cambio de visión y estrategia, por lo que se han puesto en marcha campañas y herramientas para aumentar la valoración positiva sobre dichas institución.

En el marco de esta iniciativa se enmarca la creación de las televisiones parlamentarias, televisiones que tienen como finalidad la difusión de los actos públicos generados por la institución, donde pueden observarse las sesiones parlamentarias así como entrevistas u otro tipo de información relativa sobre políticos, grupos parlamentarios y actividades de difusión de la entidad.

La televisión parlamentaria no deja de ser un mecanismo de transparencia informativa, un formato por el cual se ofrece la imagen de que todo se puede conocer sin intermediarios, sin que haya ningún tipo de manipulación, por lo que tendremos la información de primera mano, directamente de los políticos.

Independientemente de las lecturas e interpretaciones, e incluso de la veracidad, del hecho de que ésta sea una forma de transparencia y no otra mera forma de manipulación, las televisiones parlamentarias cuentan con un escaso visionado por monótonas, de escaso interés, además de tener un formato poco ágil.

Un elemento que igualmente se desea introducir es la necesidad de contar con herramientas de conservación, almacenamiento y evidentemente difusión de dicha información audiovisual. Es necesario el control del patrimonio audiovisual de un pueblo, y controlar el patrimonio por medio de una correcta selección de la información (Caldera y Arranz, 2013). Seguramente no es necesario la conservación de todo el material generado por las televisiones parlamentarias (habría que estudiar en cada una de los parlamentos los tipos documentales generados) pero sí que es importante conserva la información que será el reflejo político de una sociedad en un momento determinado.

Aunque este estudio no va a ser motivo del análisis de la gestión documental de dicha información, sí que se analiza formas de acceso y existencia de elementos que a priori nos ayudarán a conservar el material, tales como la existencia de videotecas y sus posibilidades de recuperación. 
Política y televisión. Televisión y Documentación. Documentación y preservación Patrimonial. Todo ello nos ayudará a conservar material que posteriormente pueda ser analizado por investigadores de diferentes áreas de conocimiento que podrán analizar en un futuro a nuestros dirigentes políticos desde muy diversos prismas.

\section{TELEVISIONES PARLAMENTARIAS: ESTADO DE LA CUESTIÓN}

En sus inicios la televisión fue creada para informar, formar y entretener. Desde su aparición hasta hoy la televisión ha evolucionado y se ha transformado de la mano de la evolución social y de las revoluciones tecnológicas. Desde la aparición de la televisión en España en el año 1956 son muchos y variados los formatos que se nos han ido presentado, generando multitud de canales en diversos formatos. Televisiones generalistas, autonómicas, públicas, privadas, especializadas, locales, etc. Y todas ellas han convivido en ciertos periodos de tiempo.

En la década de los ochente se produjeron una serie de cambios que marcarían la historia de la televisión. Comenzó con la firma del estatuto (más tarde reconvertido en ley) de Radio y Televisiones. Aparecieron la primera generación de televisiones autonómicas que constituyeron la FORTA (Federación de Televisiones Autonómicas), con EITB, TV3, TVGa, Canal Sur y Tele Madrid.

Al final de los años ochenta y comienzos de los noventa aparecen en el paranorama televisivo nacional las televisiones privadas con cobertura nacional: Antena 3, Telecinco y Canal +. Como consecuencia la programación televisiva comenzó a avanzar y variar en su programación, adaptándose a las nuevas exigencias.

A comienzos del S. XXI, la televisión sufre cambios drásticos con la aparición de la TDT. Aparecen canales nuevos y más especializados con las necesidades de las audiencias. De esta forma, se produce un cambio de programas generalistas a un tipo de emisiones más específica en diferentes producciones. La televisión empieza a ser un medio para entretener a las audiencias que se concentran delante de ella.

A ello, deberemos sumarle, el avance y, por supuesto, el cambio en la manera de entender y ver la televisión. Ahora podemos ver cualquier programa, serie o producto audiovisual a través de un dispositivo móvil, smartphone gracias a Internet. Lo que se conoce como televisión a la carta. En este ámbito, la información ha ido adaptándose a esta novedosa forma de divulgación. La prensa tradicional opera en Internet con ediciones digitales, la televisión ha encontrado una plataforma digital basada en el marketing, y la radio emite tanto en frecuencia analógica como en Internet toda su programación. De manera más austera lo va haciendo la información política.

Los informativos han sido, de forma general, los principales emisores de información política o, por lo menos, los primeros en llegar a los ciudadanos. En el marco democrático en el que nos encontramos, la actualidad política inunda los medios de comunicación a diario. Los ciudadanos se encuentran más recelosos ante dicho bombardeo, ya que, sienten que la clase política no les identifica. La desconfianza ante ellos va en 
aumento, por lo que las Instituciones políticas y la que la conforman empiezan a optar por alternativas que demuestren, según sus criterios, la transparencia en la información política.

$\mathrm{Si}$ agrupamos los tres conceptos nombrados anteriormente podremos definir la APPolítica. Es decir, la convergencia de los medios tradicionales, nuevos modelos de periodismo y, por supuesto, Internet. El ciudadano puede disponer en el momento que desee, cualquier tipo de información con la actualidad e inmediatez que las aplicaciones móviles nos ofrecen en la actualidad.

Camina de forma paralela a la APPolítica, el nacimiento en España, de las televisiones parlamentarias en las distintas Comunidades Autónomas, como en el Congreso de los Disputados y el Senado.

Su origen nació de la idea, preestablecida, de la televisión como servicio público, de manera que como contraprestación obtendría una rentabilidad social. También se podría considerar una manera "clara" de democratizar la información, además de crear un archivo histórico y documental de las Instituciones.

Pero, podemos asegurar, que el auge de estas televisiones parlamentarias viene marcado fuertemente por la actual crisis política. Con ello, se pretende llegar de manera más cercana al ciudadano.

Sesenta países del mundo cuentan con retrasmisión en sus parlamentos, ya sea en audio o video. Se ha apreciado una emergencia de nuevas televisiones parlamentarias, con el aumento de la ultilización de Internet. Más de veinte congresos tienen un canal estructurado, equipos, presupuesto y contenidos.

Cada modelo de televisión parlamentaria tiene características específicas propias del país en el que se encuentren, pero es importante resaltar cinco tendencias generales que rigen a todas ellas en cualquier lugar del mundo:

1. Canales que pertenecen a las televisiones públicas: Reino Unido, Australia, India (Cámara Alta), Alemania y Nueva Zelanda. Tienen espacios en canales públicos, pero no son exclusivos.

2. Canales operados y controlados por las cámaras con apoyo de proveedores externos: México, Brasil, Venezuela, Francia, República Checa, Colombia, India (Cámara Baja).

3. Canales operados y controlados por empresas u organizaciones privadas: EE.UU, Canadá y Suecia.

4. Canales con señal, pero que emiten eventualmente y lo hacen por Internet: España, Alemania, Italia (Senado), Chile, Nueva Zelanda, Israel.

5. Canales con señal ocasional en línea: Argentina, Perú, Italia (Diputados), Holanda, Polonia, Parlamento Europeo.

Sus inicios fueron en Australia y Nueva Zelanda (1936). Aunque el modelo de referencia en todo el mundo es el estadounidense: C-SPAN, creado en 1979. Otro de los países pioneros en televisiones parlamentarias, fue Canadá. Utiliza un modelo privado, el CPAC, subvencionado por la industria del cable y con un objetivo claramente de servicio público. 
En los países lationoamericanos, los canales de televisión del parlamento, dependen de cámaras y del servicio en línea. Sus objetivos son la transparencia en los procesos legislativos y eliminar la imagen negativa que los ciudadanos tienen de la clase política.

Destacaremos la televisión del Senado de Brasil, es la más concreta y desarrollada del mundo. Nació en 1996 con cobertura 24 horas a través de todas las plataformas con una programación completada por espacios informativos, programas culturales y debates.

México emite desde el año 2000, de forma regular. Un año después comenzó a distribuir su emisión por canales restringidos como Sky y Direct TV. Y ya en 2005, el canal de televisión del congreso emite las 24 horas del día.

En contraprestación nos encontramos con que en Europa, las televisiones pertenecientes al parlamento son privadas aunque, confluyen en el mismo objetivo que las anteriores, mostrar un servicio público.

Cuenta con catorce canales oficiales diferenciados en tres tipos:

- Servicio público: cadenas nacionales públicas (Reino Unido)

- Pertenecen a las cámaras: Francia

- En vías de experimentación y desarrollo: canal de televisión y/o Internet (España y Portugal)

Nombraremos de manera independiente el modelo del Parlamento Europeo. Ofrece retrasmisiones en directo por Internet desde Estrasburgo y Bruselas, a través, del servicio de información audiovisual Europe By Satellite (EbS). Su señal es digital, multibilingüe, no codificada, gratuita y fácil de recibir. Sus contenidos forman parte de cadenas nacionales.

En el caso del Reino Unido, la BBC es la propietaria del canal parlamentario: BBC Parliament. Cubre en vivo y diferido todo los asuntos relacionados los actividad política y real, que acontecen en el país. A partir del año 2000, tiene un espacio dedicado en la BBC News, con el apoyo del servicio DAB.

Desde 1993, Francia cuenta con un canal de asamble nacional llamado LCP - Assemblée Nationale. Tiene una programación semanal con documentales y programación propia. En el año 2000, se creó la Public Sénat con emisión en todas las plataformas y, por supuesto, 24 horas.

En España, catorce Comunidades Autónomas, el Congreso de los Diputados y el Senado, cuentan con emisión en directo a través de Internet de las sesiones parlamentarias. Además dentro de sus páginas web encontramos archivos históricos, videotecas y hemerotecas de anteriores legislaturas. De ellas, Extremadura, La Rioja, Galicia, País Vasco, Aragón, Cataluña, Valencia, el Congreso y el Senado, poseen canales propios de televisión para su distribución y producción. 


\section{METODOLOGÍA Y OBJETIVOS}

Son pocos los estudios o investigaciones llevadas a cabo de las televisiones parlamentarias, bien sean a nivel mundial, como en el territorio español. Se conocen los datos históricos del nacimiento de dicha televisión, así como la estructura que poseen en cada uno de los países más representativos, pero en aquellas televisiones donde han sido creadas recientemente, resulta difícil acceder a la información que nos llevaría a su análisis.

Las fases de la metodología de investigación se resumen en:

a) Revisión bibliográfica. Consulta de información referencial, bibliográfica y de fuentes en línea sobre la materia.

b) Recopilación de fuentes web de televisiones parlamentarias.

c) Generación de herramienta de análisis, descrita posteriormente.

d) Trabajo de campo. Análisis de las diferentes televisiones de los parlamentos españoles.

e) Análisis y valoración de los datos.

Teniendo claro la historia de las televisiones parlamentarias, sus orígenes y los países donde podemos encontrarlas, centraremos nuestro análisis en aquellas que pertenecen a las Comunidades Autónomas del territorio español. Para ello, hemos indagado en la información que disponemos. Teniendo en cuenta, que todas operan de forma online, es decir, su plataforma de difusión es Internet, nos hemos dirigido a los sitios web de los parlamentos autónomos:

- http://www.congreso.es/portal/page/portal/Congreso/Congreso/CongresoTV/ConDir

- http://www.es.parlamentodegalicia.es/sitios/web/default.aspx

- http://www.jgpa.es/portal.do

- http://www.parlamento-cantabria.es/

- http://www.parlamentovasco.euskolegebiltzarra.org/eu

- http://www.parlamentodenavarra.es/inicio/videoteca.aspx

- http://www.cortesaragon.es/

- http://www.parlament.cat/web

- http://www.cortsvalencianes.es/cs/Satellite/Layout/Page/1260974710668/Home.html?\&lang=c a_VA

- http://www.asambleamurcia.es/ 
- http://www.parlamentodeandalucia.es/webdinamica/portal-web-parlamento/inicio.do

- http://www.asambleaex.es/

- http://www.cortesclm.es/index.htm

- http://www.ccyl.es/export/sites/ccyl/index.html

- http://www.parlamento-larioja.org/

- http://www.asambleamadrid.es/ES/default.aspx

- http://www.senado.es/web/index.html

Una vez localizadas las páginas web de los parlamentos, analizaremos distintas cuestiones para realizar el análisis de contenidos (Caldera, 2013). A continuación, se muestran los aspectos tenidos en cuenta en cada una de las páginas web, así como en las opciones que nos muestran (en el caso que posean) las televisiones parlamentarias.

Se ha tenido en cuenta las siguientes características:

- Emisión directo-online. Afirmamos que la emisión de información se hace online porque la plataforma utilizada es Internet, además es en directo porque se realiza a tiempo real de las actividades que vemos. Es decir, todas las actividades parlamentarias (plenos, comisiones, aprobaciones de reformas, etc. ), son retrasmitidas por un equipo de realización con cámaras estáticas en los distintos parlamentos. Ninguna de las imágenes son editadas, simplemente pasan por un montador, intentando de esta manera que la información sea lo más transparente posible.

- Archivo histórico. Es lógico que en todos los parlamentos autonómicos exista un archivo en el que están recogidos todos los documentos de las diferentes actividades que allí se han ido recogiendo a lo largo de la historia del mismo. Están diferenciados en documentos históricos, documentación periódica y, en algunos casos, documentos especiales.

- Emisión sólo en actos parlamentarios. Como podremos ver más adelante tan sólo dos parlamentos cuentan con programación fuera de las emisiones en directo. El resto, sólo mantiene en línea la grabación cuando en ese preciso instante se están desarrollando actos.

- Videoteca. Estamos hablando en todo momento de emisiones de video, con lo cual, los parlamentos también poseen un archivo del mismo. Es decir, podremos acceder a cualquier video que haya sido emitido a través de la plataforma de Internet para visionarlo en diferido cuando se necesite.

- Hemeroteca. Recoge todos los documentos de prensa que hayan sido publicados, y en los que, por supuesto, la información contenida esté relacionada con el parlamento que nos ocupa, bien sea por algunos de sus miembros o porque el desarrollo del acto haya transcendencido a un medios de comunicación.

- Programación semanal. Todos los parlamentos cuentan con una agenda mensual de los actos o actividades que se suceden en él. En ellas, además, podemos consultar su hora de emisión. En el caso que haya producciones propias, existe una programación aparte de las emisiones que se llevarán a cabo en este tiempo en el que no hay actos oficiales.

- Canal propio. La gran mayoría de los parlamentos se ha beneficiado de la plataforma de 
Internet. La creación de canales de distribución propios es más sencilla y, por supuesto, más económica. Sus canales son sencillos (exceptuando Cataluña y el Congreso de los Diputados), nos disponen de una interfaz complicada, ni difícil de manejar. Pero si bien no debemos obviar que su accesibilidad, en algunos casos, no es práctica.

Todos los aspectos anteriormente nombrados están recogidos en la mayoría de las páginas web de los parlamentos autonómicos. Evidentemente, no todos poseen la misma relevancia ni la misma importancia de cara a una información más transparente y accesible para el ciudadano.

Si nuestro objetivo es analizar las televisiones parlamentarias en la red, la característica que tendría mayor importancia, sería la retrasmisión en directo-online, ya que, es de la única manera que la información política de esa Comunidad Autónoma pueda llegar a los ciudadanos sin haber pasado ningún filtro periodístico. Con lo cual es contradictorio, que una comunidad como el Principado de Asturias sea la única que no se beneficie de esta plataforma.

Teniendo en cuenta los modelos de países exteriores, también deberíamos recalcar la importancia de poseer un canal propio en el que no sólo mostremos tal y como están sucediendo los actos o actividades, sino que además utilicemos el entretenimiento para lanzar al ciudadano la información política.

\section{ANÁLISIS DE RESULTADOS}

Una vez recogidos todos los datos relevantes de cada una de las páginas web analizadas, deberemos tener claro cuáles han sido nuestros resultados y si nuestro objetivo (establecer una organización de la situación de las televisiones parlamentarias en España) ha sido óptimo.

Aunque la historia de dichas televisiones y su emisión en Internet son de reciente generación, todas confluyen en emisiones en directo-online a través de sus páginas web, anteriormente nombradas. Sólo habría que destacar el caso de Asturias, que no posee retrasmisión de sus actividades parlamentarias.

Además, una característica común a todas es el archivo histórico, en el que encontramos documentos relacionados con las actividades parlamentarias. Según la historia de las Comunidades Autónomas variarán en su extensión. Ligado al archivo, encontraremos la hemeroteca. En ella podemos encontrar cualquier publicación de prensa ligado a la historia del parlamento. Cabe destacar, que Andalucía es la única Comunidad Autónoma que en estos momentos y en su página web no dispone de una hemeroteca oficial.

Como consecuencia de los avances tecnológicos y de las retrasmisiones de los actos parlamentarios, algunas Comunidades comenzaron a organizar sus videos en videotecas. De manera que podremos encontrar todos los videos registrados por fecha y tipo. Tan sólo País Vasco se retrasa en la ultilización de videotecas.

No todas las Comunidades poseen un canal de retrasmisión propio a través de Internet, para los actos parlamentarios e incluso para la fabricación de contenidos audiovisuales. Asturias, Cantabria, Castilla - La Mancha y Castilla y León, son las únicas que en la actualidad retrasmiten a través de Internet pero no poseen un canal de distribución propio y, por lo tanto, no tienen contenidos adicionales. Todas las emisiones se hacen exclusivamente cuando existen actos parlamentarios pero en el caso del Congreso de los Diputados y 
Cataluña, mantienen la retrasmisión de diferentes productos audiovisuales cuando no están emitiendo actividades propias del parlamento.

La televisión parlamentaria que se enmarca de forma más negativa por su poca adaptación a la plataforma de Internet y la escasa información que se dispone, es la correspondiente al Principado de Asturias. En la otra punta de lanza se sitúa el parlamento catalán, el cual cuenta con tres canales de producción propia. Además distribuye reportajes y programas parlamentarios adicionales a la emisión propias de dicha televisión. Este modelo de televisión parlamentaria es el único que se aproxima al de Brasil o Reino Unido. Utilizan la televisión a través de Internet como un medio de difusión de información, no solamente como panel para disponer noticias diarias.

No hay que dejar atrás, la accesibilidad con la que nos encontramos en el momento de acceder a la web. En su página principal deberemos encontrar todas los aspectos anteriormente citados, para que de esta forma nos sea útil utilizarla dicha web. Un caso especial es el de Castilla y León. El interfaz para encontrar la retrasmisión de actos o actividades se encuentra oculta. Debemos "curiosear" a través de las diferentes ventanas para poder encontrarla. Es un gran error es tipo de fallos, ya que, al igual que en la televisión, debemos mantener la atención del usuario facilitándole la labor de búsqueda.

\begin{tabular}{|c|c|c|c|c|c|c|c|}
\hline & $\begin{array}{l}\text { Emisión } \\
\text { dir.online }\end{array}$ & $\begin{array}{l}\text { Archivo } \\
\text { histórico }\end{array}$ & $\begin{array}{c}\text { Emisión sólo en } \\
\text { actos }\end{array}$ & Videoteca & Hemeroteca & $\begin{array}{c}\text { Programación } \\
\text { semanal }\end{array}$ & $\begin{array}{l}\text { Canal } \\
\text { Propio }\end{array}$ \\
\hline Galicia & 斗 & 斗 & 为 & 幽 & 为 & & 斗 \\
\hline Asturias & & 必 & & 必 & 必 & & \\
\hline Cantabria & 为 & 斗 & 斗 & 斗 & 斗 & 斗 & \\
\hline País Vasco & 斗 & 斗 & 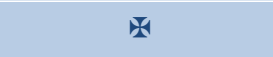 & & 头 & & 为 \\
\hline Navarra & 幽 & 必 & 斗 & 幽 & & & \\
\hline Aragón & 斗 & 斗 & 里 & 斗 & 斗 & 为 & 必 \\
\hline Cataluña & 斗 & 斗 & & 幽 & 斗 & 必 & 斗 \\
\hline Valencia & 斗 & 斗 & 头 & 斗 & & 斗 & 斗 \\
\hline Murcia & 必 & 必 & & 为 & 斗 & 斗 & \\
\hline Andalucía & 斗 & 必 & 斗 & 斗 & & 必 & 必 \\
\hline Extremadura & 斗 & 斗 & 斗 & 幽 & 斗 & 斗 & 斗 \\
\hline $\begin{array}{l}\text { Castilla- } \\
\text { LaMancha }\end{array}$ & 斗 & 斗 & 幽 & 斗 & 斗 & 头 & \\
\hline Castilla y León & 斗 & 为 & 斗 & 斗 & 斗 & & \\
\hline La Rioja & 斗 & 头 & 斗 & 幽 & 头 & 斗 & 头 \\
\hline Madrid & 斗 & 斗 & 斗 & & 斗 & 斗 & \\
\hline Congreso & 斗 & & 必 & 斗 & 必 & 必 & 必 \\
\hline Senado & 斗 & 必 & 斗 & 斗 & 头 & 斗 & 斗 \\
\hline
\end{tabular}

Tabla 1. Características televisiones parlamentarias en sus páginas web 
A la vista de la Tabla 1, que resume y sistematiza el trabajo realizado en el trabajo de campo, pudiera parecer que existe una unanimidad en la forma de hacer y entender la televisión parlamentaria, aunque tal y como se ha podido describir en reflexiones anteriores, existen claras diferencias en técnicas de difusión como en las maneras de producción del material audiovisual de las televisiones parlamentarias.

No obstante, sí que se aprecia un importante esfuerzo y despliegue informativo por parte de los gabinetes de comunicación de las diferentes asambleas y parlamentos autonómicos con el fin de poner a disposición del ciudadano la información audiovisual generada por parte de las instituciones públicas.

La crisis política y la crisis institucional se traduce en intentos de cercanía y muy especialmente de elementos que incorporen transparencia a la labor política.

Aunque el trabajo de campo no se ha centrado en el análisis de la gestión documental de la información audiovisual en los parlamentos, y que será motivo de otro trabajo posterior, sí que puede señalarse una realidad a la vista de una primera cata: la información audiovisual en las televisiones parlamentarias cuenta con escasa gestión documental que potencial la salvaguarda del Patrimonio Audiovisual de un territorio o pueblo determinado (Caldera y Arranz, 2012), por lo que hemos de señalar que muchas de estas colecciones están en grave problema de subsistencia y de accesibilidad retrospectiva por parte de investigadores, de la sociedad, o de la ciudadanía en general.

\section{CONCLUSIONES}

Recordando el pasado histórico de las televisiones parlamentarias en el mundo, es sencillo recordar de qué manera tan especialmente estructurada se encontraban en EE.UU, Brasil o Reino Unido. Con ello, y haciendo comparativa (aun no siendo justa por los años de ventaja que nos sobrepasan), la televisión parlamentaria en España se encuentra en una fase beta.

No ha conseguido alcanzar el mayor de sus objetivos, una trasparencia política y de información para optimizar la opinión de los ciudadanos. Su accesibilidad en varios casos es, todavía, nula.

Es difícil encontrar la retrasmisión de los actos, no son páginas fáciles de entender de forma intuitiva. Ergonómicamente son muy mejorables, sin lugar a dudas.

$\mathrm{Ni}$ por parte del Gobierno Central, ni por el de las Comunidades propias existe una distribución de información acerca de este servicio. Es decir, el conocimiento de estas televisiones parlamentarias es escasa por parte de la ciudadanía. Es incomprensible el que se realicen grandes esfuerzos por poner en marcha estas televisiones y sin embargo no sean publicitadas, siendo por tanto poco conocidas y poco accesibles. En algunos casos parece que es más una existencia estética y obligada más que decisión integrada y asimilada por la propia institución.

Debemos darnos cuenta que la información política es indispensable en el día a día de nuestros ciudadanos, y que es obligación mostrarsela de una forma clara y sencilla. Y dicha accesibilidad aún hoy debe ser trabajada para que sea realmente accesible. 


\section{REFERENCIAS BIBLIOGRÁFICAS}

Alcudia Borreguero, Mario; Legorburu Hortelano, José María; Barceló Ugarte, Teresa (coord.). Convergencia de medios. Nuevos desafíos para una comunicación global. Madrid: CEU Ediciones, 15 de agosto de 2012, pp. $357-374$.

Caldera-Serrano, Jorge (2013). Metodología para el análisis de repositorio institucional de colecciones audiovisuales digitales // Documentación de las Ciencias de la Información, 36, pp. 209-219.

Caldera-Serrano, J (2014) Resumiendo documentos audiovisuales televisivos: propuesta metodológica // Perspectivas em Ciência da Informação, v.19, n.2, p.147-158.

Caldera-Serrano, Jorge; Arranz-Escacha, Pilar (2012). Documentación audiovisual en televisión. Barcelona: EPI/UOC.

Caldera-Serrano, Jorge; Arranz-Escacha, Pilar (2013). Cambios en los métodos de selección documental en los sistemas de información digital en las televisiones // Investigación Bibliotecológica, 27 /60), pp. 15-26

Navarro, Fidela \& Salas, Leticia. Televisión parlamentaria. Avances y modelos en el mundo, [en línea], [29/10/2013]. Disponible en la web: http://wikicomunicacion.org/1/113/

Moreno Díaz, Julio. La información institucional en el Senado de España a través de su canal de televisión oficial, II Congreso Internacional de Comunicación 3.0 (Salamanca), 4 y 5 de octubre de 2010, pp. $1-9$ 